\title{
A POSTERIORI IMAGE-CORRECTING "DECONVOLUTION" BY HOLOGRAPHIC FOURIER-TRANSFORM DIVISION
}

\author{
G. W. STROKE and R. G. ZECH \\ The University of Michigan. Ann Arbor. Michigan, USA
}

Received 12 June 1967

\begin{abstract}
Division of spatial field distributions in the Fourier-transform domain for "resolution-retrieving". "corrective" image deconvolution of the "smearing" resulting from extended "instrument functions" in optical imaging, spectroscopy and radio-astronomy may be accomplished by a "two-filter" holographic scheme.
\end{abstract}

It has long been known [1-8] that the availability (experimental or by computation) of the intensity distribution in the instrumental "spread" function $h(x, y)$ in the imaging domain $(x, y)$ of an optical instrument (telescope, spectrograph, spectrometer, radio-astronomy antenna, etc.) would permit to retrieve the "true" Gaussian intensity distribution $f(x, y)$ in the image from actual recordings $g(x, y)$, photographic or other, where $g(x, y)=h(x, y) \circledast f(x, y)$, and where indicates a spatial convolution [1]. "Super-resolutions" [8-10], a posteriori "sharpening" of defocused images, etc., could thus be obtained, notably in the absence of noise.

Image deconvolution may be carried out along the lines of "spatial filtering", first described by Maréchal and Croce [11], who showed that the deconvolution problem of $g(x, y)=h(x, y) \circledast f(x, y)$ could be solved in the Fourier-transform domain $(u, v)$, where we have $G(u, v)=\bar{H}(u, v) \bar{F}(u, v)$, with $T[g]=\bar{G}, T[h]=\bar{H}$ and $T[f]=\bar{F}$, with $T[]=$ $\int_{-\infty}^{+\infty} \int_{-\infty}^{+\infty}[] \exp [2 \pi \mathrm{i}(u x+v y)] \mathrm{d} x \mathrm{~d} y[1]$, using spatial Fourier-transform relations between the $\bar{E}$ fields in the pupil and the focal plane of a lens, for example, and vice-versa, under usual electromagnetic-theory approximations [e.g. 7, pp. 504 to 514]. Holograms, first described by Gabor [13], appear most suitable for realizing complex spatial filters [12].

Previously-described schemes of "spatial filtering", e.g. using holograms [13] as "matched filters" $[12,14,15,1]$, may be considered as complex-amplitude multiplication schemes, symbolized by $\bar{G}=\bar{H} \bar{F}$, resulting in a correlation (bright spot) between the image and some reference "object" sought in it $[12,15,1]$, respectively of their spatial derivatives (contour correlation) [14], and not in a "deconvolved" ("corrected", or "sharpened") image, which may be obtained by complex-amplitude division $\bar{G} / \bar{H}=\bar{F}$, as we describe here.

To obtain $\bar{F}(u, v)$ from $\bar{G}(u, v)=\bar{H}(u, v) \bar{F}(u, v)$ and from it, by inverse Fourier-transformation the image function $f(x, y)$, we may multiply $\bar{G}(u, v)$ by a function $\bar{H}^{-1}=\bar{H}^{*} /|\bar{H}|^{2}$, where ${ }^{*}$ indicates a complex conjugate [1], giving $\bar{G} \bar{H}^{-1}=\bar{H} \bar{F}=\bar{F}$, the desired result. The solution of the "deconvolution" problem reduces to that of the realization of $\bar{H}^{-1}$.

Realization of the filter $\bar{H}^{-1}$ may be achieved by separately realizing the filter "components" $|\bar{H}|^{2}$ and $\bar{H}^{*}$, where $|\bar{H}|^{2}=\bar{H} \bar{H}^{*}$, and by eventually using the two filter "components" "in series", placed one after the other, next to each other, along the optical axis in the "Fourier" domain $(u, v)$, for example.

Realization of the $|\bar{H}|^{-2}$ filter component may be accomplished simply by recording (e.g. photographically) the intensity distribution $|\bar{H}(u, v)|^{2}$ in the spatial Fourier-transform $\bar{H}(u, v)$ of $h(x, y)$, using the now usual "coherent optics" methods [1]. The transmittance $\left|T_{1}(u, v)\right|$ of the photographic plate, [in the linear portion of the Hurther and Driffield [1] curve, where the slope is $\gamma$ ], is $\left|T_{1}(u, v)\right|=C\left[|\vec{H}(u, v)|^{2} \Delta t\right]^{-\frac{1}{2} \gamma}$, where $\Delta t$ is the exposure time, and $C$ a constant [1]. Using carefully controlled photographic processing $[1,17]$ and a "gamma" $\gamma \approx 2$ (see ref. 13), we obtain for the transmittance of the $|H|^{-2}$ filter component the function $\left|T_{1}(u, v)\right| \approx[C / \Delta t] /|\bar{H}|^{2}$.

Realization of the $\vec{H}^{*}$ filter component may be achieved by recording a Fourier-transform hologram of $h(x, y)$, on a photographic emulsion, for 
example, i.e. by recording $\left[\bar{H}(u, v)+\bar{E}_{\mathbf{R}}(u, v)\right] \times$ $\left[\bar{H}(u, v)+\bar{E}_{\mathrm{R}}(u, v)\right]^{*}$, where $\bar{E}_{\mathrm{R}}(u, v)$ is a coherentbackground field [1]. The intensity distribution $I(u, v) \Delta t$ in the hologram is $I(u, v) \Delta t=|\bar{H}|^{2}+$ $\left|\bar{E}_{\mathrm{R}}\right|^{2}+\bar{H} \bar{E}_{\mathrm{R}}^{*}+\bar{H}^{*} \bar{E}_{\mathrm{R}}$ (where suitable timeaveraging is implied, where appropriate, as throughout the rest of this paper). The corresponding transmittance is found [1] to be equal to $\left|T_{2}(u, v)\right|_{-} \approx 1-\frac{1}{2} \gamma I(u, v) \Delta t$, which we may consider to be in the form of a photographic "negative". The transmittance of the corresponding photographic "positive" [which may be obtained by "printing" [13] from $\left.\left|T_{2}(u, v)\right|_{-}\right]$may be made equal to $\left|T_{2}(u, v)\right|_{+}=1-\left|T_{2}(u, v)\right|=$ $=\frac{1}{2} \gamma I(u, v) \Delta l$, with suitable photographic precautions. It follows that the transmittance of the $\bar{H}^{*}$ filter component is equal to $\left|T_{2}(u, v)\right|_{+}=\frac{1}{2} \gamma \Delta t\left[|\bar{H}|^{2}+\right.$ $\left.\left|\bar{E}_{\mathrm{R}}\right|^{2}+\bar{H} \bar{E}_{\mathrm{R}}^{*}+\bar{H}^{*} \bar{E}_{\mathrm{R}}\right]$.

It is clear that the "in series" association of the two filter components $\left|T_{1}\right|$ and $\left|T_{2}\right|+$ in the form $\left|T_{1}\right|\left|T_{2}\right|_{+}$will indeed produce a "filtering" term equal to $\bar{H}^{-1}=\bar{H}^{*} /|\bar{H}|^{2}$, and that transmission of the field $\bar{H} \bar{F}$ through the "in-series" filter will result in the field $F$ in one of the holographic "side bands" [1]. Noting that $\bar{E}_{R}=\left|\bar{E}_{R}\right| \exp i \varphi_{R}$, where $\varphi_{R}$ is the linear "off-set" phase shift [1] used in the recording of the Fourier-transform hologram of $\bar{H}$, and assuming that the magnitude of the reference-background field is $\left|\bar{E}_{\mathrm{R}}\right|=2 / \mathrm{C}$, we find that the last term, $\frac{1}{2} \mathrm{C} \gamma \bar{E}_{\mathrm{R}} \bar{F}$, of the field transmitted through the "in-series" filter is indeed an angularly separated, "deconvolved" wave $\bar{F}(u, v)$, of which the Fourier transform is the desired "perfect" Gaussian image $f(x, y)$. Experimental verifications are under way.

There exist obvious relations of this work to the methods of a posteriori apodization, which are being investigated by Jacquinot and RoizenDossier, and others [18], and we note obvious applications to other "coherent optics" imageprocessing methods $[1,16]$. One of us (GWS) wishes to especially acknowledge many fruitful comments and private communications from Professor Dennis Gabor with regard to this work, and we also wish to acknowledge fruitful discussions with Professor F. T. Haddock, Dr.J. P. Wild and T. E. Graedel regarding radio-astronomical applications. The kind support of the Office of Naval Research and of the National Aeronautics and Space Administration of parts of this work is also acknowledged with much gratitude.

\section{References}

1. For a general background, definitions and notations, see e.g. G.W.Stroke, An introduction to coherent optics and holography (Academic Press. New York, 1966).

2. J.Arsac. Optica Acta 6 (1959) 103.

3. P. Jacquinot, Rep. Progr. Physics. 23 (1960) 267.

4. G.W.Stroke, Rev. Optique 39 (1960) 290.

5. R. N. Bracewell, I.R.E. Trans. A P-9, No. 1 (1961) 49.

6. J.P. Wild, Proc. Roy. Soc. (London). A 262 (1961) 84.

7. G.W.Stroke, Diffraction gratings in Handbuch der Physik, Vol. 29, ed. S. Flügge (Springer Verlag, Berlin and Heidelberg, 1967).

8. G. Toraldo di Francia, La diffrazione della luce (Edizione Scientifiche Einaudi, publ. Paolo Boringhieri, Torino, 1958).

9. J.C.Simon, G. Broussaud and E.Spitz, Comptes Rend. 248 (1959) 2309.

10. A.I. Kartashev. Optika i spektroscopiya 9 (1960) 204 (English translation).

11. A. Marechal and P. Croce. Comptes Rend. 237 (1953) 607.

12. A. Vander Lugt, I.E.E.E. Trans. Infor. Theory 10 (1964) 139.

13. D. Gabor, Nature 161 (1948) 777; Proc. Roy. Soc. (London) A 197 (1949) 454; Proc. Phys. Soc. (London) 64 (1951) 449.

14. S. Lowenthal and Y.Belvaux, Comptes Rend. 262 (1966) 413.

15. J.Ch. Viénot, J. Bulabois and G. Perin. Comptes Rend. 263 (1966) 1300.

16. D.Gabor, G.W.Stroke, R. Restrick, A. Funkhouser and D. Brumm, Physics Letters 18 (1965) 116.

17. A.A. Friesem, A.Kozma and G.F.Adams, Applied Optics 6 (1967) 851.

18. P. Jacquinot and B. Roizen-Dossier, Apodisation in Progress in Optics 3, ed. E. Wolf (North-Holland Publ. Co., Amsterdam, 1964). 